\title{
Control de Generación de Aerosoles y Cuidados Contra el SARS-CoV-2 en los Consultorios Odontológicos
}

\author{
Control of Aerosol Generation and Care Against SARS-CoV-2 in Dental Offices
}

\author{
Alexandra Portugal Infantas ${ }^{1}$; Britto Ebert Falcón Guerrero ${ }^{2}$ \& Gladys Karina Portugal Motocanche ${ }^{3}$
}

PORTUGAL, I. A.; FALCóN, G. B. E. \& PORTUGAL, M. G. K. Control de generación de aerosoles y cuidados contra el SARS-CoV-2 en los consultorios odontológicos. Int. J. Odontostomat., 15(2):330-334, 2021.

RESUMEN: Durante la atención odontológica se generan aerosoles infecciosos que aumentan el riesgo de contagio con SARS-CoV-2, de ahí que es importante mejorar las medidas de protección personal. El objetivo es dar a conocer el potencial de riesgo que tiene la generación de aerosoles infecciosos y los cuidados que se deben tener en el consultorio odontológico. se realizó una búsqueda informatizada utilizando información científica disponible a través de bases de datos como PubMed y Cochrane library, con los siguientes términos médicos (MeSH): "dental aerosol transmission", " SARS-CoV-2" y " COVID-19". el control de aerosoles es fundamental para evitar la transmisión aérea y disminuir la concentración de gotitas infecciosas en un medio relativamente cerrado y sin buena ventilación. El uso apropiado del equipo de protección personal desempeña un papel importante para reducir la contaminación, a estos equipos de protección se le ha sumado como complemento dispositivos de barrera para contener los aerosoles, aunque su uso masivo aún no ha sido validado con la evidencia científica. Conclusiones: esta revisión es una contribución global para adaptarse a la demanda de prevención ante la pandemia COVID-19, resaltando los cuidados a considerar para prevenir la infección por la presencia de aerosoles infecciosos dentro del trabajo odontológico.

PALABRAS CLAVE: dental, SARS-CoV-2, COVID-19, aerosoles, transmisión, control de infecciones, equipo de protección personal.

\section{INTRODUCCIÓN}

La Organización Mundial de la Salud (OMS) nombró a la nueva neumonía viral como enfermedad del coronavirus (COVID-19), cuyo agente causal es el coronavirus del síndrome respiratorio agudo severo-2 (SARS-CoV-2) (Guo et al., 2020), que es un bcoronavirus con ARN pleomórfico (subgénero sarbecovirus, subfamilia Orthocoronavirinae) caracterizado por una alta velocidad de recombinación genética debido a errores constantes en su proceso de replicación dependiente de la ARN polimerasa (RdRP); según la secuencia genética del virus, se sospecha que el murciélago es el huésped natural que origina este virus, (Chen et al., 2020; Pereira et al., 2020; Cheng \& Shan, 2020) por eso se pensó que la transmisión era de animal a humano; seguida de la difusión de humano a humano a través de aspirados de gotitas, heces y por aerosoles (Lai et al., 2020).
Gracias a la afinidad del coronavirus con el receptor 2 de la enzima convertidora de angiotensina (ACE2), que se encuentra en la mucosa oral y el tracto respiratorio inferior (Lo Giudice, 2020), se ha identificado el SARS-CoV-2 dentro de la saliva en estado replicativo y transmisible, reforzando el hecho de la transmisión a través del contacto con gotas al hablar, toser, estornudar y de aerosoles generados durante los procedimientos odontológicos; donde la cercanía a la cara y nariz del paciente, la exposición frecuente a saliva y sangre, y el manejo de instrumentos punzocortantes aumentan el riesgo de trasmisión (Xu et al., 2020). Ante esta situación, la Administración de Salud y Seguridad Ocupacional (OSHA.gov) clasificó a los odontólogos en la categoría de muy alto riesgo de infección debido a la exposición al coronavirus a través de procedimientos que generan aerosoles que pue-

\footnotetext{
1 Escuela Profesional de Odontología, Universidad Privada de Tacna, Tacna, Perú

${ }^{2}$ Facultad de Odontología, Universidad Latinoamericana CIMA, Tacna, Perú.

${ }^{3}$ Escuela Profesional de Odontología, Universidad Privada de Tacna, Tacna, Perú.
} 
den viajar distancias largas y permanecer en el aire, contaminando distintas superficies por mayor tiempo, siendo necesario contar con protocolos estrictos y efectivos de bioseguridad y el uso apropiado de equipo de protección personal (EPP), para reducir significativamente el riesgo de transmisión viral (lyer et al., 2020; Zhang et al., 2020), protegiendo la salud y seguridad de los odontólogos ante el riesgo de contaminación cruzada en la práctica clínica durante la fase activa de la actual pandemia de COVID-19 (Chen et al.; Martins-Filho et al., 2020).

El personal de salud odontológico juega un papel importante para prevenir la transmisión de SARSCoV-2 dentro de su ámbito de trabajo, de allí la importancia de realizar esta revisión, con el objetivo de dar a conocer el potencial de riesgo de la generación de aerosoles infecciosos y los cuidados que hay que tener dentro del consultorio odontológico.

\section{MATERIAL Y MÉTODO}

Se realizó una búsqueda informatizada desde enero de 2020 hasta el 23 de Octubre de 2020, con la cual se elaboró una revisión bibliográfica con el objetivo de describir aspectos del potencial de riesgo de la generación de aerosoles infecciosos y los cuidados que hay que tener dentro del consultorio odontológico, utilizando información científica disponible a través de bases de datos como PubMed y Cochrane library.

Como criterios de inclusión se tuvo que las fuentes bibliográficas resultaran de documentos provenientes de revistas científicas arbitradas con procesos de revisión por pares y publicadas en idioma inglés.

Los criterios de exclusión fueron, descartar todos los artículos que el resultado de la búsqueda no brindara acceso al texto completo o no tenga un código DOI que permitiera ubicar el artículo en su fuente primigenia. Se utilizaron descriptores a partir de los siguientes encabezados de términos médicos (MeSH), para elaborar las estrategias de búsqueda: "dental aerosol transmission", " SARS-CoV-2" y " COVID-19".

Después de realizada la estrategia de búsqueda, se encontraron inicialmente un total de 54 trabajos de los cuales, una vez excluidos los inadecuados según los criterios prestablecidos, se seleccionaron 31 para el desarrollo del tema investigado.

\section{RESULTADOS}

Aerosoles y su importancia en la transmisión de enfermedades. El aerosol es un sistema de dispersión coloidal en el que las partículas sólidas o líquidas se dispersan y suspenden en un medio gaseoso. Los aerosoles infecciosos son microorganismos, como bacterias, virus y micoplasmas, que se unen a partículas de aerosol y se suspenden en el aire; originándose al estornudar, toser, hablar, cantar y reír (Samaranayake et al., 2020; Pasnick et al., 2020).

La distancia y el tiempo que las partículas permanecen suspendidas en el aire está determinada por el tamaño de las partículas, la velocidad de sedimentación, la humedad relativa y el flujo de aire, y se definen como: gotas respiratorias: son partículas grandes de agua que se depositan rápidamente en una superficie después de producirse. Se definen como de $>5$ $\mathrm{mm}$ de diámetro y pueden llegar hasta por 1 metro de distancia permaneciendo en el aire por períodos de tiempo más cortos que los aerosoles; y aerosoles respiratorios: partículas $<5 \mathrm{~mm}$ que permanecen suspendidas en el aire durante minutos $u$ horas y crean un aerosol que tiene una capacidad de difusión superior a 1 metro de distancia y pueden llegar a propagarse a través de sistemas de ventilación (Lo et al.; Pasnick et al.).

Debido a la gravedad, las gotas más grandes caen al suelo rápidamente; por lo tanto, la transmisión por gotitas requiere una estrecha proximidad física entre un individuo infectado y uno susceptible. Por otro lado, las gotas o partículas pequeñas evaporadas tienen una velocidad de sedimentación baja, por lo que pueden permanecer en el aire por más tiempo y viajar hasta por seis pies de distancia (Ge et al., 2020).

Por tal razón, la práctica odontológica es considerada siempre de riesgo, ya que es una generadora frecuente de aerosoles que contienen agentes potencialmente infecciosos, como aquellos encontrados en la sangre, la saliva y la cavidad oral del paciente; debiendo prevenir el contacto de las manos con superficies contaminadas para evitar la adquisición y transferencia de patógenos a los ojos, la nariz o la cavidad oral (Ge et al.; Khader et al., 2020).

Control de la generación de aerosoles en el consultorio odontológico. El objetivo del control de la generación de aerosoles es evitar la transmisión aérea y disminuir la concentración de gotitas infecciosas 
en el medio ambiente, por el potencial que tienen de permanecer en el aire durante un tiempo considerable en un ambiente relativamente cerrado y sin buena ventilación (Ge et al.; Yue, 2020), en tal razón, el odontólogo enfrenta un riesgo particular de infección por SARS-CoV-2 gracias al contacto cercano con los pacientes y la posible exposición a gotitas contaminadas con saliva y aerosoles generados durante los procedimientos dentales, y a la vez las superficies que resulten contaminadas con saliva podrían provocar una posible infección cruzada (Li et al., 2020).

De manera que, el control de la transmisión relacionada con aerosoles en la clínica dental es fundamental, siendo recomendable seguir las siguientes recomendaciones: (1) La actividad dental debe limitarse a tratamientos que no se pueden posponer o de urgencia y se sugiere organizar el flujo de pacientes para no tener más de 1 paciente en la sala de espera ( $\sin$ parejas, ni familiares); donde el paciente siempre debe usar mascarilla quirúrgica y deben evitarse los artículos personales. (2) Los pacientes que tengan signos y síntomas de gripe o resfrío, deben usar mascarillas en la sala de espera para reducir la descarga de gotitas y aerosoles patógenos; asegurándose de que se cubran la nariz y la cavidad oral con un pañuelo de papel o el antebrazo al toser o estornudar y que desechen los pañuelos usados en un cubo de basura inmediatamente después de su uso. (3) La sala de espera debe tener una ventilación natural de $60 \mathrm{~L} / \mathrm{s}$ y debe mantenerse una separación entre pacientes de al menos 1 metro. (4) Usar siempre enjuagues bucales, el uso de clorhexidina, aceites esenciales y cloruro de cetilpiridinio, reducen un $68,4 \%$ de las colonias bacterianas en aerosoles dentales; pero hay que considerar que la clorhexidina no parece ser eficaz ante el SARS-CoV-2. Es recomendable usar agentes oxidantes como enjuagues que contengan peróxido de hidrógeno al $1 \%$ o yodopovidona al 0,2 \% para reducir la carga viral en la saliva, en particular, cuando no se usa el dique de goma. También se considera que el cloruro de cetilpiridinio $(0,05 \%$ a $0,10 \%)$ puede disminuir la gravedad del COVID-19 en sujetos infectados, reduciendo el riesgo de transmisión en la vida normal, o en aerosoles, producidos durante procedimientos dentales. (5) El uso de dique de goma reduce significativamente las partículas en el aire en un $70 \%$ dentro de 1 metro del rango de operación; y se debe usar con una succión de volumen extra alto para aerosoles y salpicaduras. (6) Evitar el uso constante del equipo de succión de saliva. (7) Evite las radiografías intraorales, debido a que estimulan la salivación y la tos, contribuyendo a la formación de aerosoles y la propagación de material biológico infectado, prefiera las radiografías extraorales como panorámicas o tomografías. (8) Evite usar la jeringa triple en su forma de spray, y prefiera el secado con algodón. (9) Evitar el uso de piezas de mano de alta velocidad sin válvulas anti-retracción para prevenir el riesgo de aspiración de virus y bacterias en los tubos de aire y agua, lo que podría causar una infección cruzada por la contaminación de la unidad dental; aparte, el aerosol emitido por una pieza de alta velocidad, alcanza un radio de hasta 2 metros de distancia. (10) Use una buena posición de trabajo para aumentar la distancia con el paciente durante los tratamientos, reduzca el tiempo de operación e indique al paciente que evite hablar tanto como sea posible. (11) Usar purificadores de aire y/o radiación ultravioleta, dado que el SARS-CoV-2 es sensible a los rayos ultravioleta y al calor, y puede inactivarse a una temperatura de $56^{\circ} \mathrm{C}$ durante $30 \mathrm{~min}$ (Ge et al.; Khader et al.; Peng et al., 2020; Gugnani \& Gugnani, 2020; Ali \& Raja, 2020; Izzetti et al., 2020; Herrera et al., 2020).

Uso de EPP contra SARS-CoV-2 en el consultorio odontológico. La necesidad de atención en salud oral durante la pandemia de COVID-19 requiere incrementar las medidas de protección en la atención odontológica, lo que demanda la elaboración de protocolos y alternativas clínicas que ayuden a prevenir y disminuir el riesgo de contagio del virus SARS-CoV-2 (Cochrane Oral Health, 2020).

Los cuidados de los odontólogos a los aerosoles infecciosos van de la mano con el uso de EPP para reducir el riesgo de exposición; antes de usar estos medios de protección, se debe iniciar con un estricto lavado de manos que es una forma simple, efectiva y de bajo costo contra la transmisión cruzada de este nuevo coronavirus. Se debenlavar, antes y después de contactar al paciente, después de la exposición a fluidos corporales y después de tocar el entorno del paciente; teniendo en cuenta que el uso de guantes no reemplaza el lavado de manos, siendo necesario lavar o desinfectar las manos después de quitarse los guantes (Ali \& Raja; Lotfinejad et al., 2020; Long et al., 2020). En caso de contaminación con sangre y material biológico, es obligatorio lavarse las manos con la técnica de lavado quirúrgico con agua corriente y jabón, complementando con el uso de soluciones desinfectantes alcohólicas (Amato et al., 2020).

Se debe utilizar antes de cada tratamiento un EPP que incluya lentes de protección ocular y mascaras faciales para proteger las membranas mucosas 
de los ojos, la nariz y la cavidad oral durante procedimientos que generen aerosoles contaminados (los pacientes también deben usar lentes de protección), usar batas desechables de aislamiento total, guantes, gorro y mascarilla quirúrgica N95 que es de ajuste hermético y tiene una eficiencia de filtrado de partículas del $95 \%$ o superior para un tamaño medio de partícula de $0.3 \mu \mathrm{m}$ (las mascarillas deben usarse una vez y desecharse) (Peng et al.; Ali \& Raja; Amato et al.; Umer et al., 2020).

Aunque estos EPP protegen las membranas mucosas a la exposición de gotas de saliva, la sangre $\mathrm{u}$ otros fluidos corporales del paciente, no parecen ser suficientes contra la inhalación de agentes infecciosos transportados por el aire (Peng et al.; Chia et al., 2020).

Haciéndose necesario contar con nuevos medios que contengan mejor los aerosoles dentales para ampliar el espectro de protección de profesionales y pacientes, logrando brindar una atención segura y de calidad dentro del consultorio odontológico (Verbeek et al., 2020).

En un esfuerzo por reducir la exposición potencial a los aerosoles generados durante el tratamiento oral, se han desarrollado sistemas transparentes de barrera para aislamiento, como cajas para aerosol, cortinas de plástico y sistemas de protección de policarbonato diseñados para cubrir la cabeza y la parte superior del cuerpo del paciente, que brindan: protección contra gotas y aerosoles, efectividad, seguridad, ergonomía y limpieza/eliminación; sin embargo, pueden aumentar la complejidad de los procedimientos. Pero se debe considerar que estos dispositivos de barrera para contener los aerosoles son un complemento y no sustituyen al EPP, y su uso masivo aún debe evitarse hasta que se presenten estudios que los validen (Sorbello et al., 2020; Russell, 2020).

\section{CONCLUSIONES}

Ante el alto riesgo que representa realizar una atención odontológica, esta revisión es una contribución global para adaptarse a la demanda de prevención ante la pandemia COVID-19, resaltando los cuidados a considerar para prevenir la infección por la presencia de aerosoles infecciosos dentro del trabajo odontológico.
PORTUGAL, I. A.; FALCÓN, G. B. E. \& PORTUGAL, M. G. K. Control of aerosol generation and care against SARSCoV-2 in dental offices. Int. J. Odontostomat., 15(2):330-334, 2021.

ABSTRACT: During dental care, infectious aerosols are generated that increase the risk of contagion with SARSCoV-2, hence it is important to improve personal protection measures. Objective: to publicize the potential risk of the generation of infectious aerosols and the care that must be taken in the dental office. Method: a computerized search was carried out using scientific information available through databases such as PubMed and Cochrane library, with the following medical terms (MeSH): "dental aerosol transmission", "SARS-CoV-2" and "COVID-19 ". Results: aerosol control is essential to prevent airborne transmission and reduce the concentration of infectious droplets in a relatively closed environment without good ventilation. The proper use of personal protective equipment plays an important role in reducing contamination. Barrier devices have been added as a complement to these protective equipment to contain aerosols, although their massive use has not yet been validated with scientific evidence. Conclusions: this review is a global contribution to adapt to the demand for prevention against the COVID-19 pandemic, highlighting the care to be considered to prevent infection due to the presence of infectious aerosols in dental work.

KEY WORDS: dental, SARS-CoV-2, COVID-19, aerosols, transmission, infection control, personal protective equipment.

\section{REFERENCIAS BIBLIOGRÁFICAS}

Ali, K. \& Raja, M. Coronavirus disease 2019 (COVID-19): challenges and management of aerosol-generating procedures in dentistry. Evid. Based Dent., 21(2):44-5, 2020.

Amato, A.; Caggiano, M.; Amato, M.; Moccia, G., Capunzo, M. \& De Caro, F. Infection control in dental practice during the COVID-19 pandemic. Int. J. Environ. Res. Public Health, 17(13):4769, 2020.

Chen, Y.; Liu, Q. \& Guo, D. Emerging coronaviruses: Genome structure, replication, and pathogenesis. J. Med. Virol., 92(4):418$23,2020$.

Cheng, Z. J. \& Shan, J. 2019 novel coronavirus: where we are and what we know. Infection, 48(2):155-63, 2020.

Chia, P. Y.; Coleman, K. K.; Tan, Y. K.; Ong, S. W. X.; Gum, M.; Lau, S. K.; Lim, X. F.; Lim, A. S.; Sutjipto, S.; Lee, P. H.; et al. Detection of air and surface contamination by SARS-CoV-2 in hospital rooms of infected patients. Nat. Commun., 11(1):2800, 2020.

Cochrane Oral Health. Recommendations for the re-opening of dental services: a rapid review of international sources. COVID-19 Dental Services Evidence Review (CoDER) Working Group. 2020. Disponible en: https://oralhealth.cochrane.org/sites/ oralhealth.cochrane.org/files/public/uploads/ covid19_dental_reopening_rapid_review_13052020.pdf

Ge, Z. Y.; Yang, L. M.; Xia, J. J.; Fu, X. H. \& Zhang, Y. Z. Possible aerosol transmission of COVID-19 and special precautions in dentistry. J. Zhejiang Univ. Sci. B., 21(5):361-8, 2020. 
Gugnani, N. \& Gugnani, S. Safety protocols for dental practices in the COVID-19 era. Evid. Based Dent., 21(2):56-7, 2020.

Guo, Y. R.; Cao, Q. D.; Hong, Z. S.; Tan, Y. Y.; Chen, S. D.; Jin, H. J.; Tan, K. S.; Wang, D. Y. \& Yan, Y. The origin, transmission and clinical therapies on coronavirus disease 2019 (COVID19) outbreak - an update on the status. Mil. Med. Res., 7(1):11, 2020.

Herrera, D.; Serrano, J.; Roldán, S. \& Sanz M. Is the oral cavity relevant in SARS-CoV-2 pandemic? Clin. Oral Investig., 24(8):2925-30, 2020

Iyer, P.; Aziz, K. \& Ojcius, D. M. Impact of COVID-19 on dental education in the United States. J. Dent. Educ., 84(6):718-22, 2020.

Izzetti, R.; Nisi, M.; Gabriele, M. \& Graziani, F. COVID-19 Transmission in Dental Practice: Brief Review of Preventive Measures in Italy. J. Dent. Res., 99(9):1030-8, 2020.

Khader, Y.; Al Nsour, M.; Al-Batayneh, O. B.; Saadeh, R.; Bashier, $\mathrm{H}$.; Alfaqih, M.; et al. Dentists' awareness, perception, and attitude regarding COVID-19 and infection control: crosssectional study among Jordanian dentists. J. M. I. R. Public Health Surveill., 6(2):e18798, 2020.

Lai, C. C.; Shih, T. P.; Ko, W. C.; Tang, H. J. \& Hsueh, P. R. Severe acute respiratory syndrome coronavirus 2 (SARS-CoV-2) and coronavirus disease-2019 (COVID-19): The epidemic and the challenges. Int. J. Antimicrob. Agents, 55(3):105924, 2020.

Li, Y.; Ren, B.; Peng, X.; Hu, T.; Li, J.; Gong, T.; Tang, B.; Xu, X. \& Zhou, $X$. Saliva is a non-negligible factor in the spread of COVID19. Mol. Oral Microbiol., 35(4):141-5, 2020.

Lo Giudice, R. The Severe Acute Respiratory Syndrome Coronavirus-2 (SARS CoV-2) in dentistry. Management of biological risk in dental practice. Int. J. Environ. Res. Public Health, 17(9):3067, 2020.

Long, R. H.; Ward, T. D.; Pruett, M. E.; Coleman, J. F. \& Plaisance Jr., M. C. Modifications of emergency dental clinic protocols to combat COVID-19 transmission. Spec. Care Dent., 40(3):21926, 2020.

Lotfinejad, N.; Peters, A. \& Pittet, D. Hand hygiene and the novel coronavirus pandemic: the role of healthcare workers. J. Hosp. Infect., 105(4):776-7, 2020.

Martins-Filho, P. R.; de Gois-Santos, V. T.; Tavares, C. S. S.; de Melo, E. G. M.; do Nascimento-Júnior, E.M. \& Santos, V. S. Recommendations for a safety dental care management during SARS-CoV-2 pandemic. Rev. Panam. Salud Publica, 44:e51, 2020.

Pasnick, S.; Carlos, W. G.; De la Cruz, C. S.; Gross, J. E.; Garrison, G. \& Jamil, S. SARS-CoV-2 Transmission and the Risk of Aerosol-Generating Procedures. Am. J. Respir. Crit. Care Med., 202(4):P13-4, 2020.

Peng, X.; Xu, X.; Li, Y.; Cheng, L.; Zhou, X. \& Ren B. Transmission routes of $2019-n C o V$ and controls in dental practice. Int. J. Oral Sci., 12:9, 2020

Pereira, L. J.; Pereira, C. V.; Murata, R. M.; Pardi, V. \& PereiraDourado, S. M. Biological and social aspects of Coronavirus Disease 2019 (COVID-19) related to oral health. Braz. Oral Res., 34:e041, 2020.

Russell, C. Development of a device to reduce oropharyngeal aerosol transmission. J. Endod., 46(8):1144-8, 2020.

Samaranayake, L. P.; Fakhruddin, K. S.; Ngo, H. C.; Chang, J. W. W. \& Panduwawala, C. The effectiveness and efficacy of respiratory protective equipment (RPE) in dentistry and other health care settings: a systematic review. Acta. Odontol. Scand., 78(8):626-39, 2020

Sorbello, M.; Rosenblatt, W.; Hofmeyr, R.; Greif, R. \& Urdaneta, F. Aerosol boxes and barrier enclosures for airway management in COVID-19 patients: a scoping review and narrative synthesis. Br. J. Anaesth., 125(6):880-94, 2020.
Umer, F.; Haji, Z. \& Zafar, K. Role of respirators in controlling the spread of novel coronavirus (COVID-19) amongst dental healthcare providers: a review. Int. Endod. J., 53(8):1062-7, 2020.

Verbeek, J. H.; Rajamaki, B.; Ijaz, S.; Sauni, R.; Toomey, E.; Blackwood, B.; Tikka, J. H.; Ruotsalainen, C. \& Balci, F. S. K. Personal protective equipment for preventing highly infectious diseases due to exposure to contaminated body fluids in healthcare staff. Cochrane Database Syst. Rev., 4(4):CD011621, 2020.

Xu, R.; Cui, B.; Duan, X.; Zhang, P.; Zhou, X. \& Yuan, Q. Saliva: potential diagnostic value and transmission of 2019-nCoV. Int. J. Oral Sci., 12(1):11, 2020.

Yue, L. Ventilation in the dental clinic: an effective measure to control droplets and aerosols during the Coronavirus pandemic and beyond. Chin. J. Dent. Res., 23(2):105-7, 2020.

Zhang, Z.; Liu, S.; Xiang, M.; Li, S.; Zhao, D.; Huang, C. \& Chen, S. Protecting healthcare personnel from 2019-nCoV infection risks: lessons and suggestions. Front. Med., 14(2):229-31, 2020.

Dirección para correspondencia:

Britto Ebert Falcón-Guerrero

Av. Tarapacá \# 350

Tacna

PERÚ

E-mail: artdent2000@hotmail.com 